\title{
Trastuzumab Administration in Patients with Metastatic Breast Cancer - Experience of a Large University Breast Center
}

\author{
Verträglichkeit und klinische Praxis der Trastuzumab-Langzeitbehandlung von Patientinnen mit \\ metastasiertem Mammakarzinom - Erfahrungen aus einem großen universitären Brustzentrum
}

Authors

Affiliations
A. D. Hartkopf ${ }^{1}$, M. H. Brendel ${ }^{1}$, M. Wallwiener ${ }^{2}$, F.-A. Taran ${ }^{1}$, S. Brucker ${ }^{1}$, E.-M. Grischke ${ }^{1}$

${ }^{1}$ Department of Obstetrics and Gynecology, University of Tübingen, Tübingen

${ }^{2}$ Department of Obstetrics and Gynecology, University of Heidelberg, Heidelberg
Key words

- breast cancer

- Her-2/neu

- metastasis

- chemotherapy

- trastuzumab

Schlüsselwörter

- Mammakarzinom

- Her-2/neu

- Metastasierung

- Chemotherapie

- Trastuzumab

Deutschsprachige Zusatzinformationen online abrufbar unter: www.thieme-connect.de/ ejournals/toc/gebfra

\section{received 12.11.2013 \\ revised 16.1.2014 \\ accepted 30.1.2014}

\section{Bibliography}

DOI http://dx.doi.org/

10.1055/s-0034-1368244

Geburtsh Frauenheilk 2014; 74

563-568 @ Georg Thieme

Verlag KG Stuttgart · New York ISSN 0016-5751

\section{Correspondence}

Dr. Andreas Daniel Hartkopf,

M. D., M. Sc.

University of Tübingen

Department of Obstetrics

and Gynecology

Calwerstraße 7

72076 Tübingen

andreas.hartkopf@

med.uni-tuebingen.de

\section{Abstract \\ $\nabla$}

Background: Administered either alone or in combination with various cytostatic, endocrine or targeted therapies, trastuzumab significantly improves the prognosis of patients with HER2-positive breast cancer. As trastuzumab is effective across multiple lines of therapy in the metastatic setting (treatment beyond progression: TBP), it is often administered over a long period of time. The aim of this study was to evaluate the tolerability and clinical practice of long-term trastuzumab administration ( $>1$ year) in metastatic breast cancer patients treated in a large university breast center. Methods: Metastatic breast cancer patients who received at least 18 cycles of trastuzumab administered every three weeks at the University Gynecological Hospital of Tuebingen between 1999 and 2012 were included in this retrospective study. Typical combination drugs, side effects, and the impact of administration on left ventricular ejection fraction (LVEF) were investigated.

Results: 72 patients were eligible for inclusion in the study. The mean number of administrations was 50.14 (SD: 27.51). In 53 patients the principle of TBP was followed across an average of 2.4 therapy lines. Classic cardiac risk factors were present at the beginning of trastuzumab treatment in 34 patients (47\%). Seven patients (10\%) experienced a decrease in LVEF during treatment, 9 patients (13\%) had hypersensitivity reactions. Treatment was discontinued in two patients due to side effects $(1 \times$ progressive LVEF decrease, $1 \times$ intolerance).

Summary: The administration of trastuzumab across multiple lines of therapy was generally tolerated well. Cardiac risk factors were not a limiting factor. If regular cardiac monitoring is done, trastuzumab appears not only to improve survival but also helps preserve the quality of life of patients with HER2-positive metastatic breast cancer.

\section{Zusammenfassung \\ $\nabla$}

Hintergrund: Trastuzumab trägt sowohl als Monotherapeutikum sowie in Kombination mit verschiedenen zytostatischen, endokrinen und zielgerichteten Therapien maßgeblich zur Prognoseverbesserung von Patientinnen mit HER2positivem Mammakarzinom bei. Da Trastuzumab in der metastasierten Situation über mehrere Therapielinien hinweg wirksam ist (treatment beyond progression: TBP), wird es häufig über einen langen Zeitraum verabreicht. Ziel der vorliegenden Arbeit ist die Evaluation der Verträglichkeit und klinischen Praxis der Trastuzumab-Langzeittherapie (>1 Jahr) des metastasierten Mammakarzinoms an einem großen universitären Brustzentrum.

Methoden: In einer retrospektiven Studie wurden klinische Daten von Patientinnen erfasst, die an der Universitäts-Frauenklinik Tübingen zwischen 1999 und 2012 mindestens 18 3-wöchentliche Gaben Trastuzumab in der metastasierten Situation erhielten. Hierbei wurden insbesondere typische Kombinationspräparate, Nebenwirkungen und der Einfluss der Behandlung auf die linksventrikuläre Ejektionsfraktion (LVEF) untersucht.

Ergebnisse: 72 Patientinnen konnten in die Analyse eingeschlossen werden. Die durchschnittliche Anzahl der Applikationen betrug 50,14 (Standardabweichung: 27,51). Bei 53 Patientinnen wurde das TBP-Prinzip im Durchschnitt über 2,4 Therapielinien hinweg angewandt. Bei $34 \mathrm{~Pa}-$ tientinnen (47\%) waren zu Beginn der Trastuzumab-Therapie klassische kardiale Risikofaktoren vorhanden. Sieben Patientinnen (10\%) hatten während der Behandlung einen LVEF-Abfall, Unverträglichkeitsreaktionen fanden sich in 9 (13\%) Fällen. Die Behandlung wurde aufgrund von Nebenwirkungen bei 2 Patientinnen abgebrochen $(1 \times$ progredienter LVEF-Abfall, $1 \times$ Unverträglichkeit). 
Zusammenfassung: Die Anwendung von Trastuzumab über mehrere Therapielinien hinweg wurde insgesamt gut vertragen. Kardiale Risikofaktoren waren bei der Langzeitanwendung kein limitierender Faktor. Unter regelmäßiger kardiologischer Kontrolle scheint Trastuzumab daher nicht nur zu einem verbesserten Überleben, sondern ebenso zum Erhalt der Lebensqualität von Patientinnen mit HER2-positivem metastasiertem Mammakarzinom beizutragen.

\section{Introduction}

Breast cancer is the most common malignancy in women. Despite increasing treatment options and the availability of potentially curative therapy for primary disease, metastatic disease is still considered incurable [1]. Therapeutic approaches for metastatic disease are of a palliative nature and must be evaluated according to their potential to preserve patients' quality of life. For this reason, AGO recommends the administration of less toxic substances as first-line treatment of metastatic disease (http:// www.ago-online.de). Treatment therefore consists primarily of endocrine and targeted therapies.

Human epidermal growth factor receptor (HER2) is overexpressed in around $20 \%$ of breast cancers. HER2 overexpression is associated with more aggressive tumor growth and a poorer prognosis [2,3]. Compared to HER2-negative tumors, HER2-positive tumors occur more frequently in younger women and nodepositive women and often respond less well to cytotoxic or endocrine therapies $[4,5]$. The monoclonal antibody trastuzumab (Herceptin $^{\circledR}$ ) plays an important role in the treatment of HER2positive metastatic breast cancer. It binds to the extracellular domains of HER2-receptors, inhibiting the HER2 signaling pathway and promoting the destruction of tumor cells by the immune system $[6,7]$. Trastuzmab was approved for treatment of metastatic breast cancer by the FDA in 1998 and in Europe in 2000.

Trastuzumab is effective both as a monotherapy and in combination with other substances [8]. The current standard for first-line therapy is a combination of trastuzumab with the taxanes paclitaxel and docetaxel $[9,10]$. Synergies between trastuzmab administration and other cytostatic substances have also been reported $[11,12]$. The combination of trastuzumab with endocrine therapies results in a less toxic therapy regimen [13]. The continued administration of trastuzumab across different therapy lines (treatment beyond progression, TBP) has been shown to offer benefits for the treatment of HER2-positive metastatic breast cancer $[14,15]$.

Trastuzumab is generally well tolerated. Apart from rare allergic reactions, the most important side effect associated with trastuzumab therapy is cardiotoxicity, which manifests primarily as an asymptomatic decrease in left ventricular ejection fraction (LVEF) [16]. Cardiac dysfunction occurring with trastuzumab administration has been particularly noted in patients who had prior anthracycline-based therapy. Regular echocardiographic follow-up every three months of patients to monitor LVEF is therefore recommended during trastuzumab therapy [17]. As decreases in LVEF are usually reversible, therapy with trastuzumab can often be continued as long as cardiovascular risks are closely monitored ( $\odot$ Table $\mathbf{1}$ ).

Although approval of trastuzumab was based on large prospective studies, there is not much data on the long-term administration of trastuzumab. The aim of this study was to evaluate the tolerability and clinical practice of long-term trastuzumab adminis- tration ( $>1$ year) in metastatic breast cancer patients treated in a large university breast center.

\section{Material and Method \\ $\nabla$}

The clinical data of all female patients with metastatic breast cancer treated at the University Hospital of Tübingen between August 1999 and February 2012 who received at least 18 doses of trastuzumab were included (administration interval: 3 weeks; dosage: $6 \mathrm{mg} / \mathrm{kg}$, intravenous; loading dose: $8 \mathrm{mg} / \mathrm{kg}$ ). Treatment cycles administered in the (neo-)adjuvant situation were only counted if metastatic disease occured during or directly after (neo-)adjuvant treatment and if tratstuzumab therapy was continued for at least 18 applications in the metatstatic setting. Patients were also excluded from the study if there were no records of regular (every 3 months) follow-up with echocardiography done either by the Cardiology Department of the Medical University Hospital of Tübingen or by an external physician.

The patient's HER2 status was evaluated using the HerceptTest ${ }^{\mathrm{TM}}$ (DAKO, Glostrup, Denmark). The HerceptTest ${ }^{\mathrm{TM}}$ uses immunohistochemical staining for the semi-quantitative detection of HER2 expression, which is assessed using a scale from 0 to $3+$. A score of $3+$ conclusively indicates a HER2-positive status, while a score between 0 and $1+$ signifies that the tumor is HER2-negative. If the score is 2+, FISH test (Pathvysion ${ }^{\circledR}$ Kit, Vysis, Downers Grove, IL, USA) is used to re-evaluate HER2 amplification.

All data were recorded on Excel spreadsheets (Version 2007, Microsoft, Redmond, Washington DC, USA). Statistical evaluation was done using PASW Statistics 20 (SPSS Inc., Chicago, IL, USA). Figures were produced using Prism 5 (GraphPad Software, La Jolla, CA, USA). The measures of central tendency, mean and standard deviation were calculated for continuous variables; frequency distributions were calculated for categorical variables. Survival curves were calculated using the Kaplan-Meier method. Time from the start of trastuzumab administration till progres-

Table 1 Management of cardiac side-effects.

\begin{tabular}{|c|c|c|}
\hline LVEF & $\begin{array}{l}\text { Trastuzumab ad- } \\
\text { ministration }\end{array}$ & Echocardiography \\
\hline slightly reduced & continue therapy & every 4 weeks \\
\hline reduced to $<45 \%$ & continue therapy & $\begin{array}{l}\text { every } 2-4 \text { weeks } \\
\text { if no improvement } \\
\text { occurs: stop therapy }\end{array}$ \\
\hline $\begin{array}{l}\text { reduced to }<30 \% \\
\text { asymptomatic }\end{array}$ & $\begin{array}{l}\text { temporarily dis- } \\
\text { continue therapy }\end{array}$ & $\begin{array}{l}\text { every } 2 \text { weeks } \\
\text { if no improvement } \\
\text { occurs: stop therapy }\end{array}$ \\
\hline $\begin{array}{l}\text { reduced to }>30 \% \\
\text { symptomatic }\end{array}$ & stop therapy & \\
\hline
\end{tabular}

Modification of [33] 
sion was analyzed. If no progression occurred during the period under observation (i.e. during trastuzumab therapy), these cases were removed from analysis at the end of the period observation (i.e. at the end of trastuzumab treatment).

\section{Results}

$\nabla$

\section{Description of cohort}

A total of 72 patients were included in the study ( Table 2 ). The majority of patients (68\%) were node-positive at primary diagnosis. Tumor size at primary operation was more than $2 \mathrm{~cm}$ in the majority of patients (pT2-4, 67\%) and the tumor grade was G12 in the majority of patients (63\%). $31 \%$ of patients had primary metastasis, 56\% were ER-positive and 44\% were PR-positive.

Average age at the start of trastuzumab treatment was 59 years (standard deviation [SD]: 11 years; median: 62 years). The youngest patient at first administration was 29 years old, the oldest patient was 83 years old ( Table 2). Most patients (75\%) were post-menopausal. The average number of administrations was 50.14 ( Fig. 1), the lowest number was 18 and the highest was 149 (SD: 27.51).

Table 2 Clinical data at primary diagnosis.

\begin{tabular}{lc} 
Characteristic & n (\%) \\
Grading & \\
\hline G1-2 & $45(63)$ \\
\hline G3 & $25(35)$ \\
\hline Unknown & $2(3)$ \\
Tumor size & \\
\hline (y)pT0-1 & $22(31)$ \\
\hline (y)pT2-4 & $48(67)$ \\
\hline Unknown & $2(3)$ \\
\hline Lymph node status & \\
\hline positive & $49(68)$ \\
\hline negative & $18(25)$ \\
\hline unknown & $5(6)$ \\
\hline Metastasis & \\
\hline M0 & $45(63)$ \\
\hline M1 & $22(31)$ \\
\hline unknown & $5(6)$ \\
\hline Histology & \\
\hline IDC & $57(79)$ \\
\hline - ILC or other & $15(21)$ \\
\hline ER status & \\
\hline p positive & $40(56)$ \\
\hline - negative & $32(44)$ \\
\hline PR status & \\
\hline - positive & $32(44)$ \\
\hline - negative & $40(56)$ \\
\hline HER2 status (IHC) & \\
\hline 2+ & $16(22)$ \\
\hline & $56(78)$ \\
\hline
\end{tabular}

IDC: invasive ductal carcinoma, ILC: invasive lobular carcinoma, IHC: immunohistochemistry

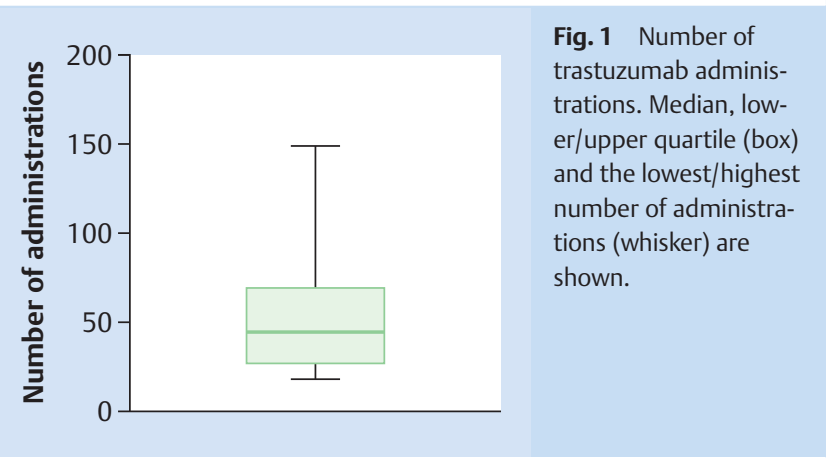

Type of initial trastuzumab therapy

Two patients (3\%) had previously received trastuzumab in the (neo-)adjuvant treatment setting ( $\bigcirc$ Table 3 ). These patients received at least 18 further doses of trastuzumab in the metastatic setting. The majority of patients (54 patients, 69\%) received trastuzumab as a first-line therapy. Trastuzumab was administered as a second-line therapy in 8 patients $(11 \%)$, as a third-line therapy in 5 patients (7\%) and as a fourth-line therapy in 3 (4\%) patients.
Table 3 Clinical data at the start of trastuzumab treatment.

\begin{tabular}{|c|c|}
\hline Age in years, mean $\pm S D$ (range) & $59 \pm 11(29-83)$ \\
\hline \multicolumn{2}{|l|}{ Menopausal status, $\mathbf{n}(\%)$} \\
\hline pre-menopausal & $9(13)$ \\
\hline post-menopausal & $54(75)$ \\
\hline not specified & $9(13)$ \\
\hline \multicolumn{2}{|l|}{ Type of initial trastuzumab therapy, n (\%) } \\
\hline (neo-)adjuvant setting & $2(3)$ \\
\hline f first line & $54(75)$ \\
\hline second line & $8(11)$ \\
\hline third line & $5(7)$ \\
\hline fourth line & $3(4)$ \\
\hline Previous anthracycline-based therapy, $\mathrm{n}(\%)$ & $42(58)$ \\
\hline Cardiac risk factors, n (\%) & $34(47)$ \\
\hline coronary heart disease & $1(1)$ \\
\hline - arterial hypertension & $24(77)$ \\
\hline - diabetes mellitus II & $6(19)$ \\
\hline - hypercholesterolemia & $7(22)$ \\
\hline nicotine abuse & $4(13)$ \\
\hline \multicolumn{2}{|l|}{ Combination therapy, $\mathbf{n}(\%)$} \\
\hline vinorelbine & $29(40)$ \\
\hline taxanes & $30(42)$ \\
\hline - capecitabine/gemcitabine & $14(19)$ \\
\hline other cytostatic agents & $11(15)$ \\
\hline - lapatinib & $6(8)$ \\
\hline pertuzumab & $1(1)$ \\
\hline - aromatase inhibitors & $30(42)$ \\
\hline Treatment beyond Progression, $\mathrm{n}(\%)$ & $53(74)$ \\
\hline across 2 therapy lines & $37(51)$ \\
\hline - across 3 therapy lines & $12(17)$ \\
\hline across 4 therapy lines & $4(6)$ \\
\hline Therapy discontinued (n) \% & 12 \\
\hline because of progressive disease & 11 \\
\hline at the patient's request & 1 \\
\hline - average duration, months (range) & $13(3-20)$ \\
\hline
\end{tabular}




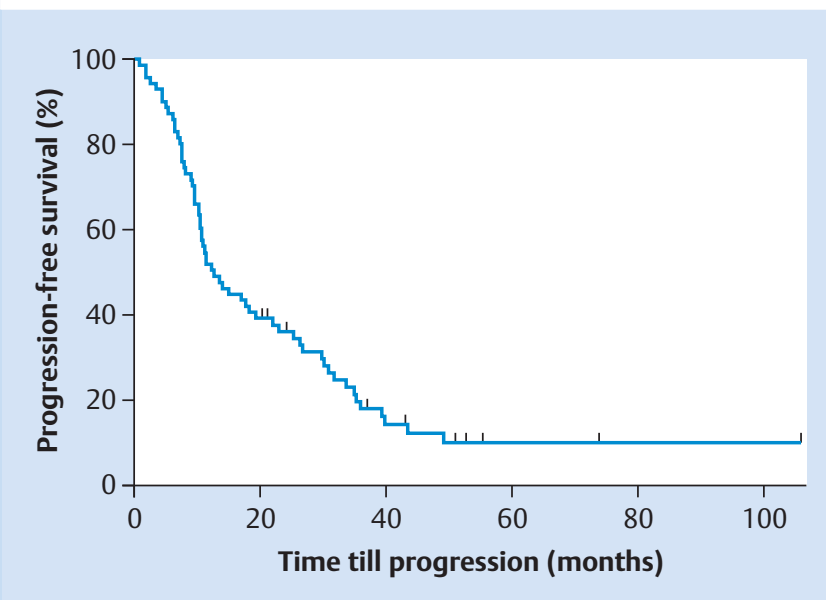

Fig. 2 Kaplan-Meier curve showing time to progression (in months) since the start of trastuzumab therapy.

\section{Previous cardiac risk factors}

Forty-two patients (58\%) had previously received anthracyclinebased treatment ( 0 Table 3 ). One patient had previous symptomatic cardiac disease (coronary heart disease). At the beginning of trastuzumab therapy, 34 patients (47\%) presented with classic cardiac risk factors (coronary heart disease, arterial hypertension, diabetes mellitus, hypercholesterolemia, nicotine abuse).

\section{Treatment beyond progression} and discontinuation of therapy

In 53 of 60 patients who experienced disease progression during trastuzumab therapy, the TBP principle was used for an average of 2.4 therapy lines ( 0 Table 3 ). The most commonly used combination agents were taxanes ( $42 \%$ of patients) and aromatase inhibitors ( $42 \%$ of patients), followed by vinorelbine ( $40 \%$ of patients) and capecitabine/gemcitabine (19\% of patients). Treatment was temporarily discontinued in 12 patients for an average of 12 months ( Table 3). Disease progression was responsible for discontinuing therapy in the large majority of these cases. In three of these patients treatment was switched to lapatinib. Median progression-free survival from the start of trastuzumab therapy was 13.44 (95\% confidence interval: 7.46-19.43) months (O Fig. 2).

\section{Side effects and termination of therapy}

Seven (10\%) patients had an asymptomatic decrease in LVEF during trastuzumab treatment ( $\bigcirc$ Table 4). In 6 of 7 cases, LVEF normalized again over the course of treatment. Only 1 patient terminated therapy because she experienced progressive decrease of LVEF with the development of symptomatic cardiac insufficiency despite pausing therapy. Interestingly, none of the patients temporarily discontinued therapy because of cardiac side effects. Nine patients (13\%) had hypersensitivity reactions with flu-like symptoms. One patient had to terminate trastuzumab therapy due to intolerance. The most common reason for terminating therapy was disease progression (22 patients, 31\%); 17 (24\%) patients terminated therapy at their own request, and $7(10 \%)$ patients died during therapy. Twenty-two patients (31\%) were still receiving treatment at the time of data analysis.
Table 4 Side effects.

$\begin{array}{ll}\text { Side effects, } \mathbf{n}(\%) & \\ \text { - LVEF decrease > } 10 \% & 7(10) \\ \text { - hypersensitivity/fever } & 9(12,5) \\ \text { Reasons for discontinuing therapy, } \mathbf{n}(\%) & \\ \text { - still receiving treatment } & 22(31) \\ \text { - conversion of therapy due to disease progression } & 22(31) \\ \text { - patient's request } & 17(24) \\ \text { - death of the patient } & 7(10) \\ \text { - unknown } & 2(3)^{*}\end{array}$

* $1 \times$ intolerance; $1 \times$ symptomatic cardiac insufficiency

\section{Discussion}

$\nabla$

The introduction of targeted therapies was an important milestone in the treatment of HER2-positive breast cancer. In 1998, trastuzumab was the first recombinant antibody drug to be approved for the treatment of metastatic breast cancer. Trastuzumab is effective as a monotherapy as well as in many different combinations with established cytostatic drugs, aromatase inhibitors and other targeted substances $[9-11,13,18]$. The wide range of combinations, the option of using trastuzumab across different therapy lines and the good tolerability of trastuzumab mean that patients with HER2-positive metastatic breast cancer often receive trastuzumab over lengthy periods of time.

The most significant side effect of trastuzumab therapy is its cardiotoxicity [9]. Depending on the therapeutic setting, the incidence of cardiotoxic side effects is between 1-27\%. The reported incidence of cardiotoxic side effects for trastuzumab monotherapy is $2-11 \%$; when trastuzumab is administered in combination with anthracyclines up to $27 \%$ of patients may experience a decrease in LVEF [19-21]. The incidence of patients experiencing a decrease in LVEF was reported to be lower in the adjuvant setting compared to the metastatic setting [22].

The decrease in LVEF is usually reversible. This means that treatment with trastuzumab can usually be continued when patients are asymptomatic and have only a limited drop in LVEF as long as patients are monitored carefully. If LVEF drops to below 30\%, trastuzumab administration should be temporarily discontinued; however, therapy can be resumed if the patient is asymptomatic and LVEF recovers [17]. In our study, treatment was only terminated for one patient due to symptomatic cardiac insufficiency; in 6 patients, the decrease in LVEF was asymptomatic and transient, which meant that treatment could be resumed.

The low rate of cardiac side effects found in our study corresponds to the data reported in the literature and appears to be due to that fact that no patient in our study received combination therapy of Herceptin with anthracyclines, as this approach is considered obsolete in the metastatic setting. However, the majority of patients had received anthracyclines prior to enrolment in our study. Moreover, more than half of our patients had no cardiac risk factors at the start of treatment with trastuzumab. The majority of these patients were older than 62 years $\left(X^{2}\right.$-test: $\mathrm{p}=0.002$, data not shown). We found no association between the existence of cardiac risk factors, prior anthracycline-based therapy or patient age and the occurrence of decreased LVEF (Fisher's exact test, data not shown). Although this finding has only limited statistical significance due to the low number of cases, it corresponds to the results of a larger study by Guarneri 
et al. [23]. Trastuzumab therapy can and should (under regular and close cardiac monitoring) therefore be also offered to older patients or patients with cardiac risk factors. Guarneri and colleagues do, however, point out that cardiac toxicity can be accompanied by an unremarkable LVEF. The use of alternative non-invasive screening tools, for example the determination of cardiac troponin and of brain natriuretic peptides, is still being evaluated in clinical trials $[22,24]$. Moreover, the selection of patients for our study was based on good tolerance a priori of trastuzumab, as one inclusion criterion was treatment duration of at least 1 year. A further limitation of our study was the lack of followup; possible long-term toxicities were not recorded.

Long-term administration of trastuzumab was well tolerated. Patients in the investigated cohort also had comparatively long progression-free survival rates. At the time of data analysis, one patient with pulmonary metastases had been receiving trastuzumab since 8 years and the course of her disease had been stable ever since. In this context, one could even say that, with trastuzumab, breast disease in this patient had become chronic. But when interpreting these data it is important to take into account that only patients who underwent treatment with trastuzumab for at least 1 year were included in the study. Overall survival was therefore per se at least 1 year.

The most common combination drugs used together with trastuzumab therapy were taxanes and vinorelbine. Compared to taxane monotherapies, a combination of paclitaxel or docetaxel and trastuzumab is associated with significantly improved survival $[10,11]$. The combination of trastuzumab and vinorelbine has been shown to be similarly effective and is additionally tolerated better [11]. AGO has already recommended such a regimen to treat HER2-positive metastatic breast cancer in early therapy lines (http://www.ago-online.de). Cytostatic therapies were generally terminated after 6 cycles or, in cases with intolerance or progression, already after 3 cycles. If there was no progression of disease, almost all patients (depending on their previous history of therapy and hormone receptor status) received either maintenance therapy combined with an aromatase inhibitor or trastuzumab administration was continued as a monotherapy. The TBP principle was used to treat patients with disease progression. Another alternative was switching to lapatinib (e.g. in combination with capecitabine), particularly to treat patients with metastases of the central nervous system.

The synergies between trastuzumab and numerous substances used in the therapy of metastatic breast cancer is the basis for the TBP principle which was followed in around two thirds of all patients. In addition to various retrospective analyses, the prospective randomized GBG 26/BIG 03-05 study was able to show for the first time that the administration of trastuzumab beyond progression resulted in longer progression-free survival times $[14,15,25]$. The authors suggest that, while trastuzumab loses its anti-proliferative effect as disease progresses, it still retains its efficacy as a chemosensitizer. The antibody-drug conjugate trastuzumab-emantasine (T-DM1) is an innovative approach which combines the synergies of HER2-targeted treatment with cytostatic therapies. It is used to selectively target HER2-overexpressing tumor cells, inhibits HER2-dependent signaling pathways and only releases the chemotherapeutic component DM1 inside the tumor cell. T-DM1 was approved for the treatment of metastatic breast cancer in 2013 based on the results of the EMILIA trial.
Another option for extensively pretreated patients consists of combining the HER2-targeting tyrosine kinase inhibitor lapatinib and trastuzumab [26]. The successful principle of a dual HER2 blockade is also achieved using the combination of trastuzumab plus pertuzumab [27]. Based on data from the CLEOPATRA trial, AGO recommends the combination therapy trastuzumab + pertuzumab + docetaxel as the new standard for first-line therapy of metastatic HER2-positive breast cancer (http://www.agoonline.de).

As the HER2 status of the primary tumor is not necessarily representative of the HER2 status of metastases [28-31], AGO recommends reevaluating the receptor status of metastatic tissue (http://www.ago-online.de). However, this is an invasive procedure which is associated with increased morbidity. In our cohort the positive HER2 status of metastatic tissue was reevaluated in 3 patients; one of these 3 patients was initially HER2-negative (data not shown). The DETECT-III trial will prospectively investigate whether the HER2 status of circulating tumor cells obtained from $7.5 \mathrm{ml}$ whole blood is suitable to predict the efficacy of HER2-targeted therapy in the metastatic setting [32].

Trastuzumab is usually administered as an intravenous infusion; a subcutaneous formulation for the administration of trastuzumab has recently been developed [33]. But this formulation was not yet available for our study. Both the efficacy and the safety profile of this new formulation appear to correspond to those of a "classic" intravenous infusion. In addition to administering a fixed dosage, the advantage of the new form of administration is that it significantly reduces the time required for administration: the injection takes around 3 to 5 minutes compared to 30-90 minutes for a standard intravenous infusion. This is the reason why patients prefer subcutaneous administration. Subcutaneous injection appears to offer a promising approach, particularly for long-term treatments and in the context of ensuring the best possible quality of life for patients [34].

\section{Conclusion}

$\nabla$

With the availability of trastuzumab therapy for HER2-positive breast cancer, the a priori poorer prognosis of these patients is now on a par with that of HER2-negative patients. The experience in our large university breast center shows that trastuzumab is well tolerated, even when administered for longer periods, and can be combined with different therapeutic options. The majority of our patients have received trastuzumab since their first-line therapy. Neither the existence of cardiac risk factors nor older patient age affected the good tolerability. Although cardiac side effects only rarely required the therapy to be terminated, regular cardiac monitoring is essential. Trastuzumab appears not only to improve survival but also contributes to improving the quality of life of patients with metastatic breast cancer. In future, subcutaneous administration of trastuzumab could further improve patients' quality of life.

\section{Conflict of Interest \\ $\nabla$}

None. 


\section{References}

1 Fasching PA, Ekici AB, Wachter DL et al. Breast cancer risk - from genetics to molecular understanding of pathogenesis. Geburtsh Frauenheilk 2013; 73: 1228-1235

2 Ross JS, Slodkowska EA, Symmans WF et al. The HER-2 receptor and breast cancer: ten years of targeted anti-HER-2 therapy and personalized medicine. Oncologist 2009; 14: 320-368

3 Sinn P, Aulmann S, Wirtz $R$ et al. Multigene assays for classification, prognosis, and prediction in breast cancer: a critical review on the background and clinical utility. Geburtsh Frauenheilk 2013; 73: 932940

4 Slamon DJ, Godolphin W, Jones LA et al. Studies of the HER-2/neu protooncogene in human breast and ovarian cancer. Science 1989; 244 : 707-712

5 Schechter AL, Stern DF, Vaidyanathan L et al. The neu oncogene: an erbB-related gene encoding a 185,000-Mr tumour antigen. Nature 1984; 312: 513-516

6 Scheuer W, Friess T, Burtscher $H$ et al. Strongly enhanced antitumor activity of trastuzumab and pertuzumab combination treatment on HER2-positive human xenograft tumor models. Cancer Res 2009; 69: 9330-9336

7 Junttila TT, Akita RW, Parsons K et al. Ligand-independent HER2/HER3/ PI3K complex is disrupted by trastuzumab and is effectively inhibited by the PI3K inhibitor GDC-0941. Cancer Cell 2009; 15: 429-440

8 Melcher C, Scholz C, Jäger B et al. Breast cancer: state of the art and new findings. Geburtsh Frauenheilk 2012; 72: 215-224

9 Slamon DJ, Leyland-Jones B, Shak S et al. Use of chemotherapy plus a monoclonal antibody against HER2 for metastatic breast cancer that overexpresses HER2. N Engl J Med 2001; 344: 783-792

10 Marty M, Cognetti F, Maraninchi D et al. Randomized phase II trial of the efficacy and safety of trastuzumab combined with docetaxel in patients with human epidermal growth factor receptor 2-positive metastatic breast cancer administered as first-line treatment: the M77001 study group. J Clin Oncol 2005; 23: 4265-4274

11 Andersson M, Lidbrink E, Bjerre Ket al. Phase III randomized study comparing docetaxel plus trastuzumab with vinorelbine plus trastuzumab as first-line therapy of metastatic or locally advanced human epidermal growth factor receptor 2-positive breast cancer: the HERNATA study. J Clin Oncol 2011; 29: 264-271

12 von Minckwitz G, du Bois A, Schmidt M et al. Trastuzumab beyond progression in human epidermal growth factor receptor 2-positive advanced breast cancer: a german breast group 26/breast international group 03-05 study. J Clin Oncol 2009; 27: 1999-2006

13 Kaufman B, Mackey JR, Clemens MR et al. Trastuzumab plus anastrozole versus anastrozole alone for the treatment of postmenopausal women with human epidermal growth factor receptor 2-positive, hormone receptor-positive metastatic breast cancer: results from the randomized phase III TAnDEM study. J Clin Oncol 2009; 27: 5529-5537

14 Gelmon KA, Mackey J, Verma S et al. Use of trastuzumab beyond disease progression: observations from a retrospective review of case histories. Clin Breast Cancer 2004; 5: 52-58; discussion 59-62

15 Tripathy D, Slamon DJ, Cobleigh M et al. Safety of treatment of metastatic breast cancer with trastuzumab beyond disease progression. J Clin Oncol 2004; 22: 1063-1070

16 Keefe DL. Trastuzumab-associated cardiotoxicity. Cancer 2002; 95 : $1592-1600$

17 Mackey JR, Clemons M, Cote MA et al. Cardiac management during adjuvant trastuzumab therapy: recommendations of the Canadian Trastuzumab Working Group. Curr Oncol 2008; 15: 24-35
18 Vogel CL, Cobleigh MA, Tripathy D et al. Efficacy and safety of trastuzumab as a single agent in first-line treatment of HER2-overexpressing metastatic breast cancer. J Clin Oncol 2002; 20: 719-726

19 Seidman A, Hudis C, Pierri MK et al. Cardiac dysfunction in the trastuzumab clinical trials experience. J Clin Oncol 2002; 20: 1215-1221

20 Russell SD, Blackwell KL, Lawrence J et al. Independent adjudication of symptomatic heart failure with the use of doxorubicin and cyclophosphamide followed by trastuzumab adjuvant therapy: a combined review of cardiac data from the National Surgical Adjuvant breast and Bowel Project B-31 and the North Central Cancer Treatment Group N9831 clinical trials. J Clin Oncol 2010; 28: 3416-3421

21 Feldman AM, Lorell BH, Reis SE. Trastuzumab in the treatment of metastatic breast cancer: anticancer therapy versus cardiotoxicity. Circulation 2000; 102: 272-274

22 Cardinale D, Colombo A, Torrisi R et al. Trastuzumab-induced cardiotoxicity: clinical and prognostic implications of troponin I evaluation. J Clin Oncol 2010; 28: 3910-3916

23 Guarneri V, Lenihan DJ, Valero V et al. Long-term cardiac tolerability of trastuzumab in metastatic breast cancer: the M.D. Anderson Cancer Center experience. J Clin Oncol 2006; 24: 4107-4115

24 Sawaya H, Sebag IA, Plana JC et al. Early detection and prediction of cardiotoxicity in chemotherapy-treated patients. Am J Cardiol 2011; 107: 1375-1380

25 Bartsch R, Wenzel C, Altorjai G et al. Capecitabine and trastuzumab in heavily pretreated metastatic breast cancer. J Clin Oncol 2007; 25: 3853-3858

26 Blackwell KL, Burstein HJ, Storniolo AM et al. Randomized study of Lapatinib alone or in combination with trastuzumab in women with ErbB2positive, trastuzumab-refractory metastatic breast cancer. J Clin Oncol 2010; 28: 1124-1130

27 Baselga J, Cortes J, Kim SB et al. Pertuzumab plus trastuzumab plus docetaxel for metastatic breast cancer. N Engl J Med 2012; 366: 109-119

28 Zidan J, Dashkovsky I, Stayerman C et al. Comparison of HER-2 overexpression in primary breast cancer and metastatic sites and its effect on biological targeting therapy of metastatic disease. Br J Cancer 2005; 93 : 552-556

29 Edgerton SM, Moore D 2nd, Merkel D et al. erbB-2 (HER-2) and breast cancer progression. Appl Immunohistochem Mol Morphol 2003; 11: 214-221

30 Gancberg D, Di Leo A, Cardoso F et al. Comparison of HER-2 status between primary breast cancer and corresponding distant metastatic sites. Ann Oncol 2002; 13: 1036-1043

31 Tanner M, Jarvinen P, Isola J. Amplification of HER-2/neu and topoisomerase IIalpha in primary and metastatic breast cancer. Cancer Res 2001; 61: $5345-5348$

32 Lux MP, Maass N, Schütz F et al. Breast cancer 2013 - interpretation of new and known data. Geburtsh Frauenheilk 2013; 73: 584-598

33 Ismael G, Hegg R, Muehlbauer S et al. Subcutaneous versus intravenous administration of (neo)adjuvant trastuzumab in patients with HER2positive, clinical stage I-III breast cancer (HannaH study): a phase 3 , open-label, multicentre, randomised trial. Lancet Oncol 2012; 13: 869-878

34 Pivot X, Gligorov J, Muller V et al. Preference for subcutaneous or intravenous administration of trastuzumab in patients with HER2-positive early breast cancer (PrefHer): an open-label randomised study. Lancet Oncol 2013; 14: 962-970 